matrix ${ }^{5}$ for time and programs (Fig. 2, top). The time matrix is arranged with "hour" columns and "five-minute" rows. To select the time of a printout and program change, a diode pin is inserted in the hole at the intersection of the desired time. Figure 2 shows diode pins installed for $6: 15,6: 35,8: 05$, and 8:30. While printout is normally required at the end of a program, subtotal printouts may be obtained by inserting a resistor diode pin in place of the diode pin, thus inhibiting the reset and stepping pulse.

A 24-position rotary solenoid switch that is connected to the columns of the program matrix is used to select the program. The position of the switch and, therefore, the program in progress is indicated by a small light at the top of each column. The individual treatments in a program appear as rows in the matrix. Each program is selected by inserting a diode pin in the hole at the intersection of the desired treatment row and program column. Shown in Fig. 2 is Treatment $C$ for Program 1 and Treatments A and B for Program 3. The treatments were described earlier. In the event that fewer than 24 programs are required for a complete cycle, a reset row appears in the matrix. A diode pin placed in any hole along the reset row will, when the rotary solenoid switch steps to that intersecting program column, cause the rotary solenoid switch to seek its home position which is Program No. 1.

\section{Shock Timer and Data Acquisition Unit}

The number of lever pulls of each monkey is totaled by separate five-decade counters. Each decade of the counters consists of a DCU, quadruple two-input AND gate, and one-half of an octuple neon light driver. The AND gates provide the means to select sequentially the DCU's four-line BCD output for printout while front panel visual readout is continuously provided by the neon lights (described earlier) from gates supplied by the neon light drivers. Decade positions on a printout line not used to display a DCU output are used to code information. For this purpose, the AND gates and neon light drivers are utilized without a DCU.

The shock, a $60-\mathrm{Hz}$ constant-current sine wave, generated from the $500 . \mathrm{V}$ rms secondary of a transformer by a relay contact closure, operates in three modes, singly or in any combination, providing for the three conditioning treatments.

The first mode triggers a shock-duration timer after an interval variable between 5 and $100 \mathrm{sec}$ unless reset by the experimental S's lever; the second delivers a continuous shock while the animal's lever is operated; and the third delivers to a shock-duration timer, triggers with asymmetric intervals having a cycle period of $100 \mathrm{~min}$. The particular sequence of the intervals are programmed via a plug-in-circuit board.

The number of shocks received by the $S$ pair in Modes 1 and 3 is counted by a two- and one-decade counter, respectively. The number of shocks received in Mode 2 is the same as the number of lever pulls of the experimental animal. To avoid the possibility of the monkeys being subjected to an undesirable number of shocks due to malfunction of the shock circuit, a latching preset shock inhibit AND gate is connected to the counter. When the preset number of shocks during any one treatment program is delivered to the Ss, the gate inhibits all further shocks and provides a visual alarm.

\section{Data Selector}

The Data Selector, upon receipt of a readout command from a Programmer, produces the following sequence of events in rapid succession ( $2.7 \mathrm{sec}$ total): Printer ac power is turned on; combinations of a readout gate and print command are sequentially sent to, respectively, the data counters and the printer; a reset pulse is sent to the counters; a stepping pulse is sent to the Programmer to initiate the next program; and Printer ac power is turned off. A 52-position telephone step-selector switch wired for self-stepped operation is used to select and generate the required gates and pulses for the readout sequence.

\section{Printer and Printout Format}

The printer is a medium-speed digital unit ${ }^{6}$ equipped for a 6-digit line capacity. Clock time occurs on the first line of the 21-line printout followed by three data lines and a blank line per programmer channel. The data lines contain the subject code, treatment code, number of paddle pulls, and number of shocks per treatment of each monkey.

\section{REFERENCES}

CLEARY, J. I. (Ed.) General Electric transistor manual. Syracuse: General Electric, 1964. P. 337.

WALSTON, J. A., \& J. R. MILLER (Eds.) Transistor circuit design. New York: McGraw-Hill, 1963.

\section{NOTES}

1. This project was supported by NIH Grants NB 04053 and MH 02895 .

2. The authors wish to acknowledge the assistance of Mr. Charles Dreyer in the completion of this project.

3. Computer Measurements Company, Model 90-512. Division of Pacific Industries, Inc., San Iernando, California.

4. A DCU costs approximately $\$ 30$. The equipment common to all channels using plastic ICs costs about $\$ 2500$, with each channel an additional $\$ 1000$. Although our original cost was $\$ 7000$, an equivalent unit consisting of three channels can be constructed for approximately $\$ 5500$.

5. Sealectro Company, 225 Hoyt Avenue, Mamaoneck, N.Y. 10543.

6. Computer Measurements Company, Model 410A. Division of Pacific Industries, Inc., San Fernando, California.

\title{
A runway for chicks
}

\section{J. DUTCH, VICTORIA UNIVERSITY OF WELLINGTON, Wellington, New Zealand}

Problems associated with using chicks in a runway are discussed. Acquisition and extinction data are presented from a sample of 12 Australorp chicks.

Chicks have been used previously as Ss in runway experiments but only with the aid of special pretraining methods that have made interspecies comparisons difficult. Grindley (1929), for example, used a prolonged successive approximation procedure and the social facilitation of a group of chicks tethered adjacent to the goal box to develop running and eating responses. These methods were necessary to overcome two major problems in the use of these animals as Ss: the length of time it takes chicks to begin eating when they are alone and their immobility when they are alone.

The feeding problem can be solved by placing chicks in groups 


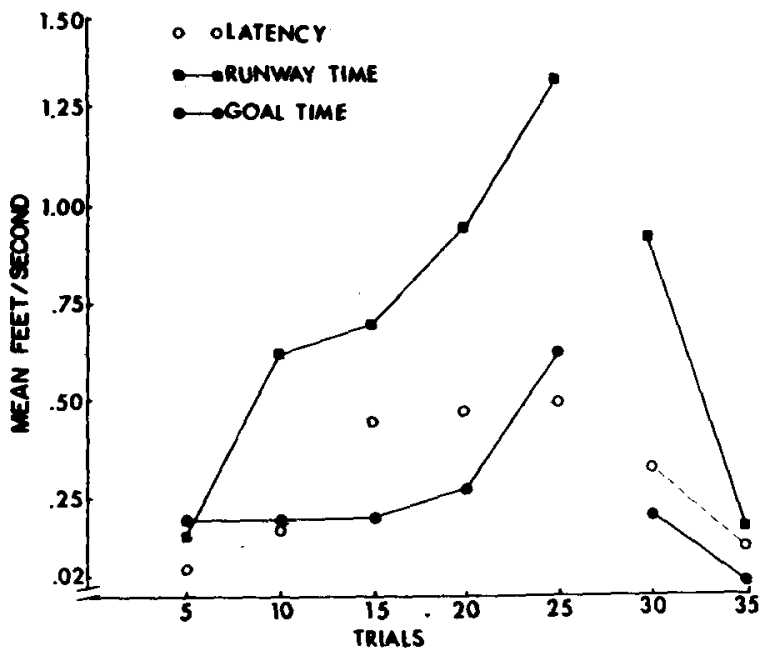

Fig. 1. Acquisition and extinction in the runway.

of four in a food-baited box for $3 \mathrm{~min}$ followed by a further placement in the box singly for $2 \mathrm{~min}$. This procedure results in immediate eating on later occasions when Ss are hungry and alone. The problem of stimulating chicks to move out of a start box was solved by light-proofing the start box and the runway and placing a lamp in or above the goal box, so that this became the only source of light. If light enters the runway from any other source it is unlikely that chicks will proceed beyond that point.

Starting at 4 days of age a limited food access of 6 in $24 \mathrm{~h}$ was sufficient to produce rapid and prolonged eating when food was made available without adversely affecting health. During deprivation is was necessary to keep illumination in the brooder to a low level to prevent cannibalism.

The runway was satisfactory for chicks ranging in age from 4 days to 4 weeks. It was made of aluminum painted flat gray with ceilings of Perspex with fitted, light-proof covers for the start box and runway. The start box measured 12 in $\times 9$ in. $H \times 8$ in. A guillotine door provided access to a runway $3 \frac{1 / 2}{\mathrm{ft}} \times 9 \mathrm{in}$. $\mathrm{H} \times 6$ in. The goal box measured 15 in. $\times 9$ in. $H \times 10$ in. and an electrically operated swing door closed behind $S$ as he entered. The food holder was an aluminum trough 3 in. $\times 2$ in. $H \times 2$ in. The food reward was Chicken Starter, a dry mixture. Ss were permitted to eat from the trough for $3 \mathrm{sec}$ at the end of each trial.

Two treadle-type microswitches in the runway and one photocell lamp in the goal box were used for triggering timers. The treadle switches consisted of two metal plates 1 in. wide with a normally open contact; when $S$ stepped on the plate the contact closed. The latency timer started when the goal box door opened and stopped when $S$ trod on the first switch situated 3 in. inside the runway. This switch also started the second timer that provided a runway measure. The second switch, 3 in. from the end of the runway, stopped the runway timer and started the goal box timer. The photocell was situated directly above the food trough so that $\mathrm{S}$ broke the beam when he placed his head into the trough.

Data were obtained with 12 Australorp chicks obtained at 1 day of age from the Wallaceville Animal Research Station, Wellington. A similar method was used with White Leghorn chicks (Dutch, 1968). The Ss were maintained in a temperaturecontrolled cage-type brooder, with food and water ad lib. After 14 days food was restricted to $6 \mathrm{~h}$ in 24 and $\mathrm{Ss}$ were handled daily for 5 days; on the 6 th day they were fed for $3 \mathrm{~min}$ in a box in groups of four. On Day 7 the procedure was repeated for individual chicks with 2-min feedings.

During acquisition each $S$ was given 5 massed trials per day for 5 days. This was followed by 10 massed extinction trials on Day 6. For the acquisition phase $S$ was placed in the start box and $5 \mathrm{sec}$ later the door was opened. The door was closed when $S$ entered the goal box. The chick was kept in the box for $4 \mathrm{sec}$ and was then carried back to the start box for the next trial. At the end of each set of daily trials, $S$ was placed in a box for $30 \mathrm{~min}$ before being returned to the brooder. The procedure for extinction was the same as for acquisition except that the food trough was empty.

Figure 1 shows the latency, runway, and goal entry times in mean feet per second. Runway time was the most sensitive measure of performance.

\section{REFERENCES}

DUTCH, J. Partial reinforcement and response reversal in chicks. Psychological Reports, 1968, 22, 272-274.

GRINDLEY, G. C. Experiments on the influence of the amount of reward on leaming in young chicks. British Journal of Psychology, 1929, 20 173-180.

\section{Measurement of electroconvulsive shock intensity}

\author{
ROBERT R. PAGANO, UNIVERSITY OF WASHINGTON, \\ Seattle, Washington 98105
}

This paper discusses the error introduced in administering ECS by calibrating intensity on the $S$ 's dc rather than ac resistance. Since the ac resistance of rats and mice can be lower than their corresponding $d c$ resistance by a factor of 50, calibrating on $d c$ resistance can introduce appreciable error depending on the current regulation of the ECS device. Examples are included to illustrate the range of possible error. In addition, an alternative method for measuring ECS intensity is presented that greatly reduces measurement error including those due to between- animal resistance differences that necessarily occur when using the usual methods.

It has come to our attention that several laboratories utilizing electroconvulsive shock (ECS) have been reporting ECS intensity based on the animal's dc resistance. However, since most researchers administer ac ( $60 \mathrm{cps}$, sinusoidal) ECS, it is the animal's ac not $\mathrm{dc}$ resistance 2 that will influence the ECS intensity. Since the ac resistance at $60 \mathrm{cps}$ is quite less than the $\mathrm{dc}$, calibrating on $\mathrm{dc}$ resistance can result in administering and reporting intensity levels that are considerably different from the actual intensity delivered. Since ECS intensity has been shown to 